\title{
Utilization of Hyperbaric Oxygen Therapy and Induced Hypothermia After Hydrogen Sulfide Exposure
}

\author{
Mir J Asif DO and Matthew C Exline MD
}

\begin{abstract}
Hydrogen sulfide is a toxic gas produced as a by-product of organic waste and many industrial processes. Hydrogen sulfide exposure symptoms may vary from mild (dizziness, headaches, nausea) to severe lactic acidosis via its inhibition of oxidative phosphorylation, leading to cardiac arrhythmias and death. Treatment is generally supportive. We report the case of a patient presenting with cardiac arrest secondary to hydrogen sulfide exposure treated with both hyperbaric oxygen therapy and therapeutic hypothermia to achieve full neurologic recovery. Key words: hydrogen sulfide; hyperbaric oxygen; therapeutic hypothermia. [Respir Care 2012;57(2):307-310. (C) 2012 Daedalus Enterprises]
\end{abstract}

\section{Introduction}

Hydrogen sulfide $\left(\mathrm{H}_{2} \mathrm{~S}\right)$ is a colorless, highly flammable, and toxic gas, which is ubiquitous to the environment. Also known as dihydrogen sulfide, sulfur hydride, or hydrosulfuric acid, the compound is usually recognized by its distinctive "rotten egg" smell. It is produced as a natural by-product of decaying organic matter by anaerobic bacteria and can also be found in geologic formations, including volcanic vents and natural gas wells. ${ }^{1}$ Exposure to $\mathrm{H}_{2} \mathrm{~S}$ predominantly occurs through an inhalation route, which may lead to illness and symptoms ranging from nausea and vomiting to end organ ischemia, including cardiac arrest.

In 2007, there were an estimated 1,134 documented exposures, with a resultant 13 fatalities. ${ }^{2}$ The mainstay of treatment for $\mathrm{H}_{2} \mathrm{~S}$ exposure is supportive care, with some institutions reporting utilization of hyperbaric oxygen

The authors are affiliated with the Davis Heart and Lung Research Institute, Division of Allergy, Critical Care, Pulmonary, and Sleep Medicine, The Ohio State University, Columbus, Ohio.

Dr Exline was partly supported by National Heart, Lung, and Blood Institute grant HL095772-02. The authors have disclosed no conflicts of interest.

Correspondence: Matthew C Exline MD, 201 Davis Heart and Lung Research Institute, 473 West 12th Avenue, Columbus OH 43210. E-mail: matthew.exline@osumc.edu.

DOI: $10.4187 /$ respcare. 01038
$\left(\mathrm{HBO}_{2}\right)$ therapy with success. ${ }^{3}$ We describe the case of a 24-year-old man presenting with cardiac arrest secondary to hydrogen sulfide exposure, treated with both hyperbaric therapy as well as induced hypothermia, which to our knowledge has not previously been reported.

\section{Case Report}

A 24-year-old male oil well worker without noteworthy medical history presented to our intensive care unit 9 hours after an early morning explosion at his job site. Following the explosion, our patient went to render aid to a coworker who was closer to the blast and had collapsed. During his efforts, our patient was overcome by the fumes and also collapsed. Fellow workers on the site were unable to rescue either man until emergency medical services arrived on the scene approximately $20 \mathrm{~min}$ later to retrieve the victims. Upon retrieval by emergency medical services, our patient was unresponsive and in cardiac arrest. Prompt cardiopulmonary resuscitation was initiated and cardioversion was needed for an "unstable rhythm," but the actual rhythm was undocumented. With resuscitation and intubation the emergency medical services personnel were able to obtain a spontaneous rhythm, and he was taken to the local emergency department for further medical care. Upon initial evaluation in the emergency department the patient was noted to be unresponsive on mechanical ventilation. He also had a strong odor of rotten eggs, consistent with hydrogen sulfide exposure. The remainder of his initial examination was otherwise unremarkable except for his poor neurologic status. Initial lab work was 


\section{Hyperbaric Oxygen and Induced Hypothermia After Hydrogen Sulfide Exposure}

notable for arterial blood gas (ABG) values showing a $\mathrm{pH}$ of $7.25, \mathrm{P}_{\mathrm{CO}_{2}}$ of $40 \mathrm{~mm} \mathrm{Hg}, \mathrm{P}_{\mathrm{O}_{2}}$ of $332 \mathrm{~mm} \mathrm{Hg}$, and bicarbonate of $14 \mathrm{mmol} / \mathrm{L}$, demonstrating an acute uncompensated metabolic acidosis. His troponin was elevated at $0.27 \mathrm{ng} / \mathrm{mL}$, indicating myocardial ischemia, and an electrocardiogram showed sinus tachycardia. The remainder of his basic metabolic panel and a complete blood count were within normal limits.

After initial stabilization and decontamination by healthcare workers wearing environmental hazard gear, including N95 masks, gloves, and gowns, he was transported to our medical center for further care. He arrived 9 hours after his initial exposure. In the interim, he continued to receive respiratory support and on arrival was hemodynamically stable off vasopressor support, with ventilator settings of volume controlled continuous mandatory ventilation, rate 8 breaths $/ \mathrm{min}$, tidal volume $550 \mathrm{~mL}(6 \mathrm{~mL} / \mathrm{kg}$ IBW), PEEP $16 \mathrm{~cm} \mathrm{H} \mathrm{H}_{2} \mathrm{O}$, and $\mathrm{F}_{\mathrm{IO}_{2}}$ 1.0. Based on an adequate $\mathrm{P}_{\mathrm{O}_{2}}$, his PEEP setting was weaned rapidly to $8 \mathrm{~cm} \mathrm{H}_{2} \mathrm{O}$ prior to initiation of hyperbaric therapy. Repeat labs showed improvement in the metabolic acidosis, with an ABG showing $\mathrm{pH}$ of 7.43 and bicarbonate of $22 \mathrm{mmol} / \mathrm{L}$. Lactate was $1.2 \mathrm{mmol} / \mathrm{L}$ and troponin had increased to $1.1 \mathrm{ng} / \mathrm{mL}$. Thiosulfate levels were not checked, and, based on the delay in his arrival to our center, he was not considered a candidate for sodium or amyl nitrite therapy. His chest radiograph was remarkable for low lung volumes, but otherwise clear. A subsequent computed tomogram of the head, without contrast, was negative for an acute bleed, and there was no evidence of increased intracranial pressure.

After further assessment, the decision was made to place the patient in a hyperbaric oxygen chamber to help assist with his inhalation injury. This was initiated within one hour of arrival at our facility, 10 hours after initial injury. The patient required initiation of a propofol drip and vecuronium to maintain ventilator synchrony while being placed in a hyperbaric chamber at 3 atmospheres of oxygen for one hour, followed by 2.5 atmospheres of oxygen for an additional hour. Overall, the patient tolerated 2 hours of $\mathrm{HBO}_{2}$ therapy well, without any hemodynamic compromise. For his cardiac arrest he was still considered a good candidate for the induced hypothermia protocol, and this was initiated on arrival to the intensive care unit, approximately 14 hours after his initial injury, with a goal temperature range of $32-34^{\circ} \mathrm{C}$, for 24 hours. Associated with his hyperbaric therapy, he did have sinus bradycardia, with a heart rate between 40 and 60 beats/min. He also experienced a brief episode of hypotension, for which he was administered norepinephrine and which resolved with a fluid bolus.

Following 24 hours of therapeutic hypothermia the patient was rewarmed per protocol. He underwent his sedation holiday per protocol. His neurological exam demon- strated improvement; he was able to follow commands, and was successfully extubated on hospital day 3,50 hours following initial injury. He was discharged on hospital day 5 in stable condition, with a residual complaint of short-term memory loss. However, shortly after discharge the patient began to experience dysarthria, tunnel vision, and right arm numbness. He was admitted to the physical medicine and rehabilitation service for further care. His visual and motor functions were found to be intact, but he did have some residual cognitive deficits, including poor working memory and a mild expressive aphasia requiring out-patient therapy. During subsequent visits the patient complained of chronic headaches, but slow improvement in his cognitive function. He was subsequently lost to follow-up at our facility, approximately 6 months after his discharge.

\section{Discussion}

This case represented an uncommon situation where hydrogen sulfide poisoning required the use of both hyperbaric oxygen therapy and induced hypothermia protocol for cardiac arrest. Our patient is one of few in the literature that we are aware of who has received and survived such an insult. Most applications utilizing both these interventions have been reported in the neurology literature in severe head injury cases. ${ }^{4}$

For decades, $\mathrm{H}_{2} \mathrm{~S}$ exposure has been a concern in occupational and environmental settings such as mining, sewage processing, liquid manure processing, crude petroleum drilling, leather tanning, rubber vulcanization, and asphalt roofing. Such exposures often involve multiple victims, as in our case, including inexperienced co-workers attempting to rescue the initial victims. ${ }^{5}$ Inhalation is the predominate route of exposure, although toxicity has also been seen with skin and eye contact. ${ }^{6}$ Acute inhalation leads to rapid absorption through the alveolar-capillary membrane and clinically mimics carbon monoxide poisoning, but with greater potency. ${ }^{7}$ At the molecular level, $\mathrm{H}_{2} \mathrm{~S}$ competitively inhibits the cytochrome oxidase aa3 system, thereby disrupting oxidative phosphorylation and aerobic metabolism. ${ }^{8}$ Patients will exhibit signs of ischemia with accumulation of lactic acid, leading to metabolic acidosis. Organs most affected include the lung, brain, and heart. Acute exposures to high concentrations can lead to bronchiolitis and reactive airway disease, eventually progressing to pulmonary edema and acute respiratory distress syndrome (ARDS). ${ }^{6}$ Neurological symptoms may range from mild symptoms such as dizziness, headaches, and slight confusion, to more serious insults such as seizures, cerebral edema, and anoxia. ${ }^{8}$ Cardiac involvement most commonly seen includes arrhythmia and cardiac arrest from ischemia.

$\mathrm{H}_{2} \mathrm{~S}$ poisoning is suspected by history and clinical symptoms and confirmed by lab findings. Witnesses may give 


\section{Hyperbaric Oxygen and Induced Hypothermia After Hydrogen Sulfide Exposure}

a history of gas exposure, and patients will often have the distinct smell of rotten eggs, and classically their silver coins turn black from the creation of metallic sulfides on the surface of the coins. ${ }^{6}$ Clinical signs of exposure include headaches, confusion, agitation, lethargy, and hypoxia. Initial labs usually indicate an acute uncompensated metabolic acidosis on chemistry panel and ABG. Further testing may indicate both an elevated lactate level and an abnormally high oxygen tension on venous blood gases, due to the uncoupling of oxidative phosphorylation and decreased uptake of oxygen by the peripheral tissue. Thiosulfate levels, a metabolite from oxidation of $\mathrm{H}_{2} \mathrm{~S}$, can be checked in the urine in nonfatal cases and in serum for fatal cases. ${ }^{9}$

Treatment efforts have been directed at supportive measures to maintain adequate oxygenation and perfusion of end organs. Along with oxygen, sodium and amyl nitrite have also been shown to help in treating $\mathrm{H}_{2} \mathrm{~S}$ poisoning. Nitrite administration results in formation of methemoglobin, which has a higher affinity for $\mathrm{H}_{2} \mathrm{~S}$ than the cytochrome oxidase aa3 system. This creates competitive inhibition of the sulfide-cytochrome interaction and allows for continued aerobic metabolism. Subsequently, the hydrogen sulfide molecule becomes unbound and oxidized to less harmful metabolites. ${ }^{10}$ Literature has shown that nitrite administration should ideally be within the first $5-10 \mathrm{~min}$ of exposure at a dose of $0.33 \mathrm{~mL} / \mathrm{kg}$ of $3 \%$ sodium nitrite for maximum effectiveness.

Another treatment modality that has been suggested by a handful of case reports for hydrogen sulfide poisoning is $\mathrm{HBO}_{2}$. Currently, the Undersea and Hyperbaric Medical Society recommends $\mathrm{HBO}_{2}$ therapy for 14 conditions, including carbon monoxide poisoning, gas gangrene, severe osteomyelitis, and, most recently, acute retinal arterial occlusion. ${ }^{11}$ The Food and Drug Administration concurs with these indications, with the exception of retinal arterial occlusion, which has not been approved by the FDA at this time.

$\mathrm{HBO}_{2}$ therapy involves placing patients in either a closed mono-place (single patient, often in a recumbent position) or multiplace (multiple patients) chamber, while administering $100 \%$ pure oxygen up to 3 atmospheres. The dive pressure, duration, and number of repeated dives vary depending on indication. In the case of hydrogen sulfide toxicity there is no recommended specific treatment protocol. Most case reports cite using similar dive pressure and duration as are used to treat carbon monoxide poisoning. ${ }^{12}$

This intervention increases oxygen availability to the peripheral tissue. In individuals with normal lung function, the majority of oxygen in the blood is transported bound to hemoglobin, while dissolved oxygen accounts only for a fraction of the total oxygen carrying capacity at $0.3 \mathrm{~mL}$ per $100 \mathrm{~mL}$ of blood. However, administering 100\% oxygen at 2.5 atmospheres increases dissolved oxygen content up to $5.5 \mathrm{~mL}$ per $100 \mathrm{~mL}$ of blood, which can meet the resting oxygen requirements of peripheral tissue. ${ }^{13} \mathrm{HBO}_{2}$ may be used even in patients with normal $\mathrm{P}_{\mathrm{aO}_{2}}$ but suspected peripheral ischemia, as its use helps reduce lactate accumulation and replenishes post-ischemic adenosine triphosphate (ATP) production. ${ }^{14}$ In patients with abnormal lung function as measured by the alveolar-arterial oxygen difference, their tissue oxygenation during $\mathrm{HBO}_{2}$ therapy will improve, but often to a lesser extent than in those patients with normal lung function, indicating that they may need either higher pressures or repeated treatments to achieve the benefits seen in patients with a normal pulmonary function. ${ }^{15}$

The primary hemodynamic effects of $\mathrm{HBO}_{2}$ use include bradycardia and decreased cardiac output. Sinus bradycardia is the most well known of these effects and has been explained by oxygen-dependent and non-oxygen-dependent mechanisms. The oxygen-dependent theory proposes the decrease in heart rate is a result of high oxygen pressure on the myocardium, which leads to increased parasympathetic tone, while the non-oxygen-dependent mechanism is thought to occur as a result of decreased sympathetic cardiac stimulation. ${ }^{16}$ It is thought this bradycardia is the main reason for overall decreased cardiac output. Hyperbaric oxygen therapy effects on systemic arterial blood pressure have been more controversial, but most research confirms an increase in peripheral vascular resistance. ${ }^{16}$

Therapeutic hypothermia has been well studied over the last 2 decades as a modality used to treat neurologic injury and ischemia, usually in the context of cardiac arrest. Early studies demonstrated a clinical benefit in patients with severe brain injuries, and further research confirmed that moderate systemic hypothermia improved neurologic outcomes in brain injury. ${ }^{17,18}$ Two randomized controlled trials in Australia and Europe in 2002 showed patients who experienced out of hospital cardiac arrest had neurologic benefit when exposed to mild therapeutic hypothermia (32$34^{\circ} \mathrm{C}$ ) for 24 hours post-arrest. Therapeutic hypothermia is thought to be protective due to a reduction in cerebral oxygen consumption, and, it is postulated, a reduction in free-radical formation, intracellular acidosis, retardation of proteolytic enzymes, protection of the fluidity of lipoprotein membranes, and a reduction in excitatory neurotransmitters. ${ }^{19}$ Patients initiated on therapeutic hypothermia protocol must also be closely monitored for hemodynamic changes, including bradycardia, hypotension, and decreased cardiac output. ${ }^{20}$ Our patient met inclusion criteria for therapeutic hypothermia due to his witnessed out of hospital arrest, and was cooled according to American Heart Association guidelines. ${ }^{21}$ There are no data on the use of therapeutic hypothermia in hydrogen sulfide toxicity or poisoning with other inhibitors of oxidative phos- 


\section{Hyperbaric Oxygen and Induced Hypothermia After Hydrogen Sulfide Exposure}

phorylation such as cyanide. However, there is biologic plausibility that it would be beneficial, due to the decreased oxygen requirements associated with hypothermia and potential reduction in toxic oxygen radicals as well.

It is important to note that any conclusions based on a single case report would be premature. Though our patient suffered a prolonged period being down in the field prior to restoration of spontaneous circulation, based on his age and previous level of health, he may have recovered without either $\mathrm{HBO}_{2}$ or hypothermic therapy. The use of $\mathrm{HBO}_{2}$ and hypothermic therapy both may require additional sedation for patient comfort, which may be deleterious in a patient with otherwise impaired cognition and lead to increased time on the ventilator. Care should be taken prior to initiating these therapies on patients who do not meet established criteria. ${ }^{11,21}$

There are few case reports of the combination of therapeutic hypothermia and hyperbaric oxygen therapy in general, and no reports of the use of combined therapy for hydrogen sulfide toxicity specifically. We report a case of a young man exposed to toxic levels of hydrogen sulfide gas and subsequent cardiac arrest treated with both hyperbaric oxygen therapy and induced hypothermic protocol and discharged home with an improved neurologic outcome.

\section{REFERENCES}

1. Woodall GM, Smith RL, Granville GC. Proceedings of the Hydrogen Sulfide Health Research and Risk Assessment Symposium October 31-November 2, 2000. Inhal Toxicol 2005;17(11):593-639.

2. Bronstein AC, Spyker DA, Cantilena LR Jr, Green JL, Rumack BH, Heard SE. 2007 Annual Report of the American Association of Poison Control Centers' National Poison Data System (NPDS): 25th Annual Report. Clin Toxicol (Phila) 2008;46(10):927-1057.

3. Smilkstein MJ, Bronstein AC, Pickett HM, Rumack BH. Hyperbaric oxygen therapy for severe hydrogen sulfide poisoning. J Emerg Med 1985;3(1):27-30.

4. Clifton GL. Hypothermia and hyperbaric oxygen as treatment modalities for severe head injury. New Horiz 1995;3(3):474-478
5. Hendrickson RG, Chang A, Hamilton RJ. Co-worker fatalities from hydrogen sulfide. Am J Ind Med 2004;45(4):346-350.

6. Munday SW. Hydrogen sulfide. New York: McGraw-Hill; 2004.

7. Voigt GE, Muller P. [Experiments on the histochemical detection of hydrogen sulfide poisoning]. Acta Histochem 1955;1(6-7):223-229.

8. Nam B, Kim H, Choi Y, Lee H, Hong ES, Park JK, et al. Neurologic sequela of hydrogen sulfide poisoning. Ind Health 2004;42(1):83-87.

9. Kage S, Takekawa K, Kurosaki K, Imamura T, Kudo K. The usefulness of thiosulfate as an indicator of hydrogen sulfide poisoning: three cases. Int J Legal Med 1997;110(4):220-222.

10. Smith RP, Gosselin RE. On the mechanism of sulfide inactivation by methemoglobin. Toxicol Appl Pharmacol 1966;8(1):159-172.

11. Gesell LB. Hyperbaric oxygen therapy indications. Undersea and Hyperbaric Medical Society; 2008.

12. Belley R, Bernard N, Cote M, Paquet F, Poitras J. Hyperbaric oxygen therapy in the management of two cases of hydrogen sulfide toxicity from liquid manure. CJEM 2005;7(4):257-261.

13. Boerema I, Meyne NG, Brummelkamp WH, Bouma S, Mensch MH, Kamermans F, et al. [Life without blood]. Ned Tijdschr Geneeskd 1960;104:949-954.

14. Gill AL, Bell CN. Hyperbaric oxygen: its uses, mechanisms of action and outcomes. QJM 2004;97(7):385-395.

15. Weaver LK, Howe S. Arterial oxygen tension of patients with abnormal lungs treated with hyperbaric oxygen is greater than predicted. Chest 1994;106(4):1134-1139.

16. Mathieu D, Favory R, Collet F, Linke JC, Wattel F. Physiologic effects of hyperbaric oxygen on hemodynamics and microcirculation. In: Mathieu D, editor. Handbook on hyperbaric medicine. Dordrecht, The Netherlands: Springer; 2006:75-102.

17. Bernard SA, Jones BM, Horne MK. Clinical trial of induced hypothermia in comatose survivors of out-of-hospital cardiac arrest. Ann Emerg Med 1997;30(2):146-153.

18. Zeiner A, Holzer M, Sterz F, Behringer W, Schorkhuber W, Mullner $\mathrm{M}$, et al. Mild resuscitative hypothermia to improve neurological outcome after cardiac arrest. A clinical feasibility trial. Hypothermia After Cardiac Arrest (HACA) Study Group Stroke 2000;31(1):8694.

19. Safar PJ, Kochanek PM. Therapeutic hypothermia after cardiac arrest. N Engl J Med 2002;346(8):612-613.

20. Bergman R, Braber A, Adriaanse M, van Vugt R, Tjan DH, van Zanten AR. Haemodynamic consequences of mild therapeutic hypothermia after cardiac arrest. Eur J Anesthesiol 2010;27(4):383387.

21. 2005 American Heart Association Guidelines for Cardiopulmonary Resuscitation and emergency cardiovascular care. Circulation 2005; 112(24 Suppl):IV1-IV203. 